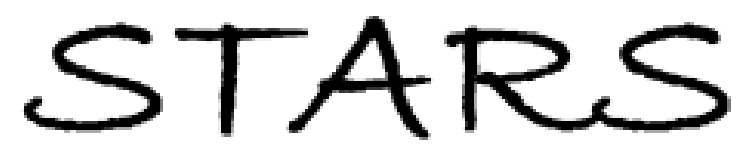

University of Central Florida

STARS

$1-1-2005$

\title{
High-contrast vertical alignment of lateral difluoro-terphenyl liquid crystals
}

Chien-Hui Wen

University of Central Florida

Sebastian Gauza

University of Central Florida

Shin-Tson Wu

University of Central Florida

Find similar works at: https://stars.library.ucf.edu/facultybib2000

University of Central Florida Libraries http://library.ucf.edu

This Article is brought to you for free and open access by the Faculty Bibliography at STARS. It has been accepted for inclusion in Faculty Bibliography 2000s by an authorized administrator of STARS. For more information, please contactSTARS@ucf.edu.

\section{Recommended Citation}

Wen, Chien-Hui; Gauza, Sebastian; and Wu, Shin-Tson, "High-contrast vertical alignment of lateral difluoroterphenyl liquid crystals" (2005). Faculty Bibliography 2000s. 4642.

https://stars.library.ucf.edu/facultybib2000/4642

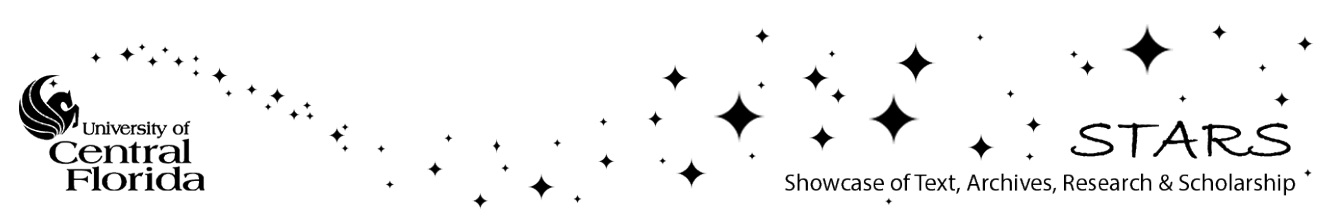




\title{
High-contrast vertical alignment of lateral difluoro-terphenyl liquid crystals
}

\author{
Chien-Hui Wen, Sebastian Gauza, and Shin-Tson Wu ${ }^{\text {a) }}$ \\ College of Optics and Photonics, University of Central Florida, Orlando, Florida 32816
}

(Received 26 May 2005; accepted 13 September 2005; published online 2 November 2005)

\begin{abstract}
A simple method for achieving high-contrast vertical alignment of lateral difluoro-terphenyl liquid crystals using buffed polyimide substrates is demonstrated. By doping a positive, nonpolar or negative dielectric anisotropic compound or mixture to the host terphenyl liquid crystals, an excellent vertical alignment is obtained. This alignment is stable throughout the entire nematic range. The selected dopants not only improve the contrast ratio but enhance the figure-of-merit of the host terphenyl mixture as well. This alignment method is particularly useful for high-contrast liquid crystal televisions. (C) 2005 American Institute of Physics. [DOI: 10.1063/1.2128068]
\end{abstract}

Vertical alignment (VA), ${ }^{1,2}$ such as multidomain VA (MVA), ${ }^{3}$ advanced superview, ${ }^{4}$ and patterned VA (PVA), ${ }^{5}$ has been widely used for direct-view and projection liquid crystal displays (LCDs) because of its unprecedented contrast ratio. To realize the useful electro-optic effects using a longitudinal electric field, the VA cells require a negative dielectric anisotropic LC mixture, i.e., $\Delta \varepsilon=\varepsilon_{\|}-\varepsilon_{\perp}<0$. For active matrix displays, high resistivity is another crucial requirement for obtaining high-voltage-holding ratio and avoiding image flickering. To achieve high resistivity, fluorinated LC compounds are commonly employed. , $^{6}$

To avoid image blurring for fast moving objects, the response time of a LCD television (TV) should be faster than $5 \mathrm{~ms}$. A straightforward method to obtain a fast response time is to reduce the LC cell gap while employing a high birefringence $(\Delta n>0.2)$ LC mixture in order to keep the necessary phase retardation. The commonly practiced onedrop-fill method enables a thin cell gap to be manufactured with high yield. To obtain a high $\Delta n$, large negative $\Delta \varepsilon$, high resistivity, and good photo and thermal stability, lateral $(2,3)$ difluoro-biphenyls and -terphenyls ${ }^{8-10}$ are the natural choices. However, these lateral difluoro-biphenyls and -terphenyls are quite difficult to align in a VA cell; a poor alignment leads to a low-contrast ratio and smeared threshold behavior.

Several VA methods have been developed. ${ }^{11-13}$ Among them, buffed polyimide (PI) and sputtered $\mathrm{SiO}_{2}$ (Refs. 14-16) are most commonly used because they can produce a stable pretilt angle. Buffed PI is preferred for large panel direct-view displays because of the low fabrication cost, while sputtered $\mathrm{SiO}_{2}$ is favored for projection displays because of its alignment uniformity. However, in both methods, it is difficult to align the lateral difluoro terphenyls. There is an urgent need to develop new methods for aligning these high $\Delta n$ difluoro compounds.

In this letter, we report a simple method for aligning the difluoro-terphenyl compounds and mixtures using buffed PI substrates. Two types of difluoro-terphenyls with molecular structures shown below were investigated:

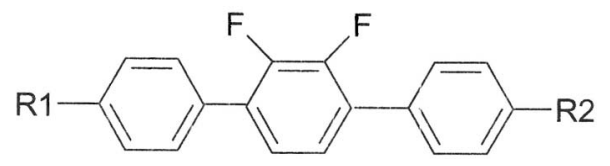

\footnotetext{
${ }^{a)}$ Electronic mail: swu@mail.ucf.edu
}

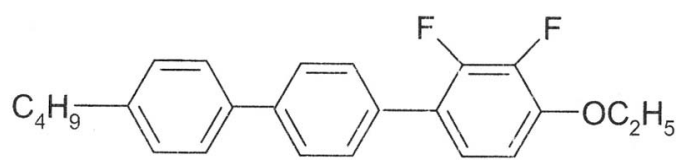

In Structure (I), two homologues with $\mathrm{R}_{1}=\mathrm{C}_{2} \mathrm{H}_{5}$ and $\mathrm{R}_{2}=\mathrm{C}_{4} \mathrm{H}_{9}$, and $\mathrm{R}_{1}=\mathrm{C}_{3} \mathrm{H}_{7}$ and $\mathrm{R}_{2}=\mathrm{C}_{5} \mathrm{H}_{11}$, were synthesized. These two homologues are pure nematics; their melting and clearing temperatures (in ${ }^{\circ} \mathrm{C}$ ) are, $[55,98]$ and $[55$, 123], respectively. To lower the melting temperature, we formulated a binary eutectic mixture, designated as Mixture A, consisting of 35:65 ratio of the -24 and -35 homologues. The nematic range of Mixture A is from 23.6 to $112.3^{\circ} \mathrm{C}$, but the supercooling effect lowers the melting point to $6.8 \mathrm{C}$. Thus, Mixture A stays liquid at room temperature $\left(\sim 23{ }^{\circ} \mathrm{C}\right)$. Compound (II) has the following phase transition sequence: Cr 96.9, Sm 117.6, N 173.8 I (in ${ }^{\circ} \mathrm{C}$ ); where $\mathrm{Cr}$, $\mathrm{Sm}, \mathrm{N}$, and I represent the crystalline, smectic, nematic, and isotropic phase, respectively. We studied the alignment characteristics of Compound (II) at an elevated temperature $(T \sim 155 \mathrm{C})$.

To prepare the PI cells, we spincoated a commercial PI SE-1211 (from Nissan Chemicals) on the indium-tin-oxide (ITO)-glass substrates and baked the substrates at $80{ }^{\circ} \mathrm{C}$ for $5 \mathrm{~min}$ and then at $180{ }^{\circ} \mathrm{C}$ for $1 \mathrm{~h}$. We then gently rubbed the ITO glass with a cloth in antiparallel directions. The rubbing induced pretilt angle is approximately $87^{\circ}$.

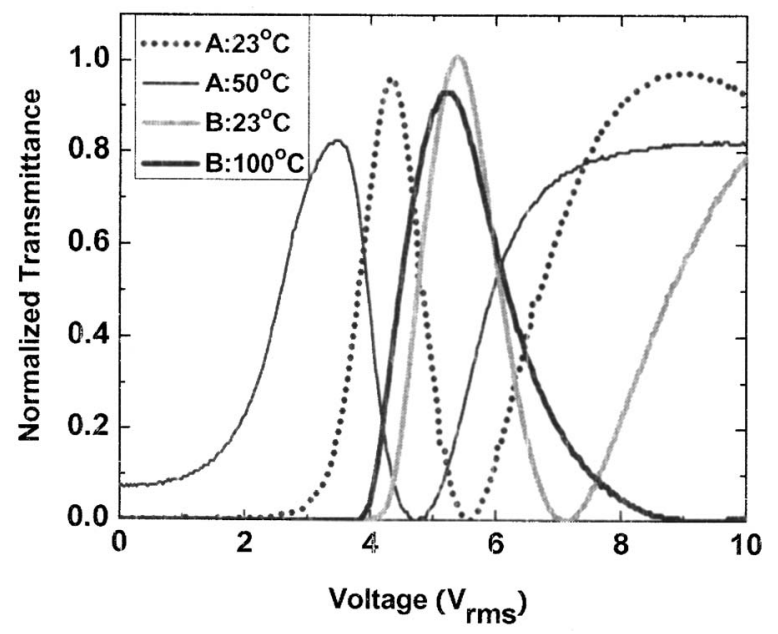

FIG. 1. VT of a VA cell between crossed polarizers at $\lambda=633 \mathrm{~nm}$. Black and dotted lines represent Mixture A at 23 and $50{ }^{\circ} \mathrm{C}$, while gray and bolded black lines represent mixture B at 23 and $100{ }^{\circ} \mathrm{C}$. 
TABLE I. Physical properties of Mixtures A, B, C, and D. $T_{m}$ : Melting temperature, $T_{c}$ : Clearing point (all in ${ }^{\circ} \mathrm{C}$ ), and $\Delta \epsilon$ : Dielectric anisotropy at $f=1 \mathrm{kHz}$.

\begin{tabular}{cccc}
\hline \hline Mixture & $T_{m}$ & $T_{c}$ & $\Delta \epsilon$ \\
\hline $\mathbf{A}$ & 23.6 & 112.3 & -1.8 \\
$\mathbf{B}$ & 21.0 & 113.1 & -1.2 \\
$\mathbf{C}$ & 16.2 & 110.2 & -1.6 \\
$\mathbf{D}$ & 6.0 & 104.7 & -2.5 \\
\hline \hline
\end{tabular}

Three types of dopants (Merck LC mixtures) were studied: MLC-9200-000, ZLI-3086, and MLC-6608. Their dielectric anisotropy $(\Delta \varepsilon)$ was $+4, \sim 0$, and -4.2 , respectively. The dopant concentration for achieving good vertical alignment depends on the $\Delta \varepsilon$ value of the dopant. Table I lists the dopant concentration and the physical properties of the four mixtures (A, B, C, and D) that we prepared for this study.

Figure 1 plots the measured voltage-dependent transmittance (VT) curves of Mixture A at $T=23$ and $50^{\circ} \mathrm{C}$. A linearly polarized $\mathrm{He}-\mathrm{Ne}$ laser $(\lambda=633 \mathrm{~nm})$ was used for these measurements. The polarizers were crossed and the on-state LC directors were oriented at $45^{\circ}$ with respect to the polarizers' optical axis. The cell gap employed is $d=5.2 \mu \mathrm{m}$, and the $\Delta n$ of Mixture A is 0.235 . Because of the large $d \Delta n$ involved, the VT curve has several transmission cycles. ${ }^{17}$ For a LCD TV, the required $d \Delta n$ should be slightly larger than $\lambda / 2$ in order to reduce the on-state voltage. From Fig. 1, mixture A has a fairly good dark state at $T \sim 23{ }^{\circ} \mathrm{C}$. The threshold voltage is $\sim 3 V_{\text {rms }}$. However, as the temperature is increased to $50{ }^{\circ} \mathrm{C}$, the light leakage at $V=0$ becomes noticeable, the threshold is smeared, and the maximum transmittance is lower than that at $T=23^{\circ} \mathrm{C}$. These phenomena imply that the pretilt angle of the VA cell is significantly increased as the temperature increases. Thus, the buffed PI cell does not provide a stable alignment for Mixture A at an elevated temperature.

The VT curves of Mixture B at $T=23$ and $100{ }^{\circ} \mathrm{C}$ were also included in Fig. 1. Mixture B is Mixture A doped with 10\% MLC-9200-000. From Fig. 1, Mixture B exhibits an excellent dark state at both temperatures. The clearing temperature of Mixture B is $113^{\circ} \mathrm{C}$. Thus, we can say that the PI cell provides a stable VA alignment in the entire nematic

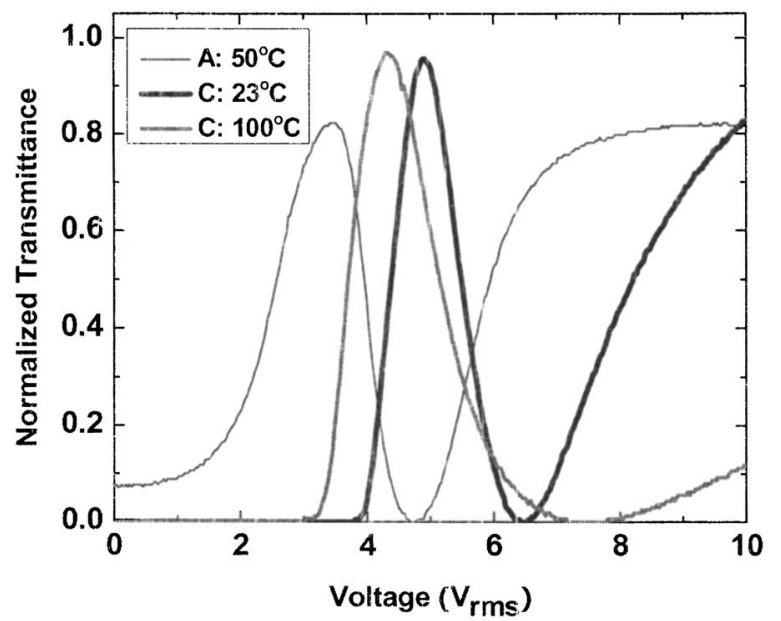

FIG. 2. VT of Mixture $\mathrm{C}$ at $\lambda=633 \mathrm{~nm}$. The data of Mixture $\mathrm{A}$ at $T=50^{\circ} \mathrm{C}$ are included as a benchmark for comparison.

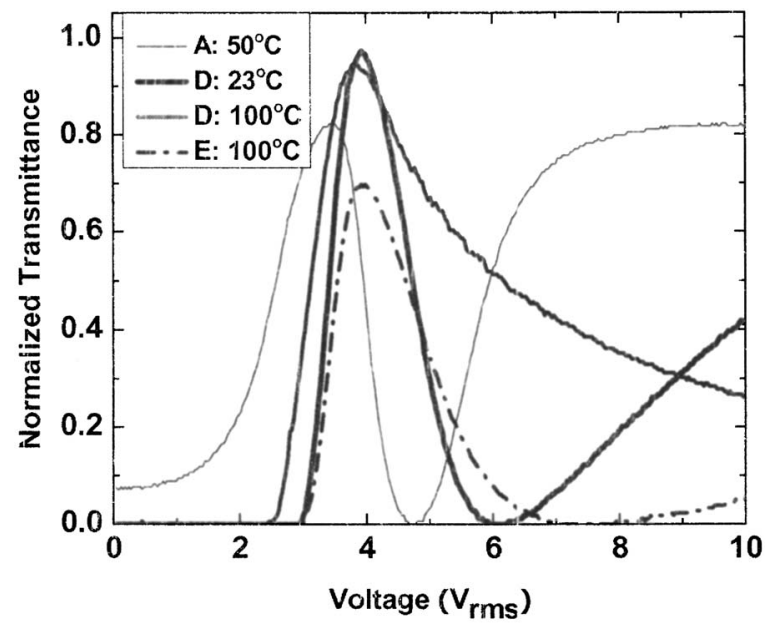

FIG. 3. VT of Mixtures D and E at $\lambda=633 \mathrm{~nm}$. The data of Mixture A at $T=50{ }^{\circ} \mathrm{C}$ are included as a benchmark for comparison.

range. The threshold voltage of Mixture $\mathrm{B}$ is $\sim 4 V_{\mathrm{rms}}$ at $23{ }^{\circ} \mathrm{C}$, which is higher than that of the host Mixture $\mathrm{A}\left(V_{\text {th }}\right.$ $\left.\sim 3 V_{\text {rms }}\right)$. This is because the positive $\Delta \varepsilon$ dopant MLC-9200000 lowers the $\Delta \varepsilon$ of Mixture B, as shown in Table I. At an elevated temperature, the threshold voltage of Mixture B slightly decreases because the bend elastic constant $(K)$ decreases more quickly than the dielectric anisotropy $(\Delta \varepsilon){ }^{6}$

Mixture C contains $10 \mathrm{wt} \%$ of ZLI-3086, a nonpolar LC mixture. Figure 2 shows the VT curves of Mixture $\mathrm{C}$ at 23 and $100{ }^{\circ} \mathrm{C}$. In both temperatures, an excellent dark state is obtained and, moreover, the threshold is sharp. Mixture C has a lower threshold voltage than Mixture B. This is because dopant ZLI-3086 is basically nonpolar so that its $\Delta \varepsilon \sim 0$. As shown in Table I, the $\Delta \varepsilon$ of Mixture $\mathrm{C}$ is -1.6 .

The required dopant concentration for improving the alignment capability depends on the physical property of the dopant employed. ZLI-3086 is a nonpolar mixture and MLC9200-000 is a positive $\Delta \varepsilon$ LC mixture consisting of axially fluorinated compounds. From Table I, adding $\sim 10 \mathrm{wt} \%$ of this type of mixture to the host mixture dramatically improves the dark state of the VA cell. On the other hand, MLC-6608 is a negative $\Delta \varepsilon$ LC mixture which already consists of $\sim 50-60 \%$ lateral difluoro-cyclohexane-phenyl compounds. Thus, the required concentration for seeing a

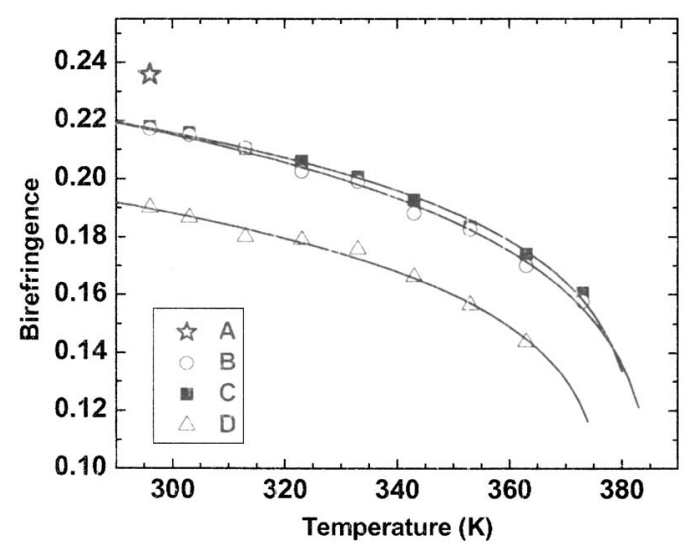

FIG. 4. Temperature-dependent birefringence of Mixtures B, C, and D. Circles, squares, and triangles are measured data for Mixtures B, C, and D, respectively. Star represents the measured data of Mixture A. Solid lines are fitting results using Eq. (1) at $\lambda=633 \mathrm{~nm}$. 


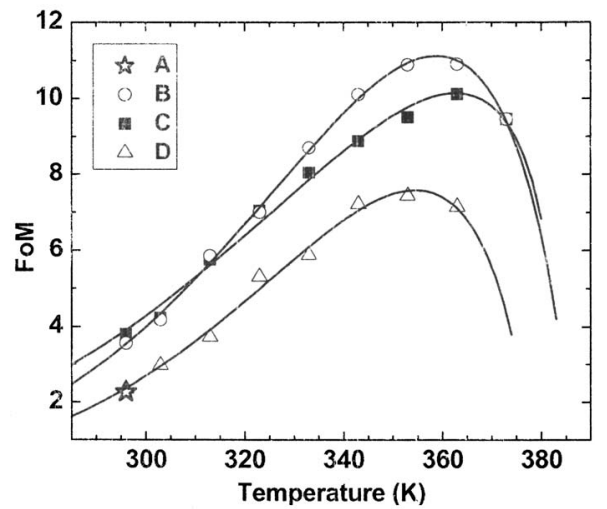

FIG. 5. Temperature-dependent FOM (in units of $\mathrm{ms} / \mu \mathrm{m}^{2}$ ) of Mixtures B, C, and D. Circles, squares, and triangles are measured data for Mixtures B, C, and D, respectively. Star represents the measure data of Mixture A. Solid lines are fitting results using Eq. (2) at $\lambda=633 \mathrm{~nm}$.

noticeable improvement is higher. This effect is demonstrated in Fig. 3 where Mixture D contains 30 wt $\%$ of MLC-6608, while Mixture E contains only 13\%. Although Mixture E exhibits a good dark state at $V=0$, its maximum transmittance in a voltage-on state only reaches $\sim 70 \%$ indicating that its alignment quality is still imperfect at elevated temperatures. Increasing the MLC-6608 concentration to $30 \%$ leads to excellent dark and bright states and a sharp threshold.

In addition to enabling excellent molecular alignment, doping $10 \%$ of a positive or nonpolar LC mixture leads to a higher figure-of-merit (FOM) which is defined as $\mathrm{FOM}=K_{33}(\Delta n)^{2} / \gamma_{1}$. Figures 4 and 5 depict the measured temperature-dependent birefringence and FOM, respectively, for Mixtures B, C, and D at $\lambda=633 \mathrm{~nm}$. Circles, squares, and triangles represent the measured data of Mixtures B, $\mathrm{C}$, and D, respectively. The birefringence of Mixture A at $T=23{ }^{\circ} \mathrm{C}$ is included in Fig. 4 as a benchmark for comparison. The solid lines represent the fitting curves using the following equations: ${ }^{18}$

$$
\begin{aligned}
& \Delta n=(\Delta n)_{o}\left(1-T / T_{c}\right)^{\beta}, \\
& \mathrm{FOM} \sim(\Delta n)_{o}^{2}\left(1-T / T_{c}\right)^{3 \beta} \exp (-E / k T) .
\end{aligned}
$$

In Eq. (1), $(\Delta n)_{o}$ is the birefringence at $T=0 \mathrm{~K}, E$ is the activation energy of the rotational viscosity, $k$ is the Boltzmann constant, and exponent $\beta \sim 0.25$ is not too sensitive to the LC structure. Since the birefringence $(\Delta n \sim 0.08)$ of all the three dopants we studied is much smaller than that of Mixture A $(\Delta n \sim 0.235)$, Mixture D has the smallest birefringence among all the mixtures studied, as shown in Fig. 4, because of its highest dopant concentration.

The host difluoro-terphenyl mixture has a higher viscosity than the three dopants employed. Thus, the final mixtures' viscosity is reduced. This explains why Mixtures B and $\mathrm{C}$ have a $\sim 2 \times$ higher figure-of-merit than the host Mixture $\mathrm{A}$ at room temperature. Because of the lower $\Delta \mathrm{n}$ of Mixture D, its FOM is lower than that of Mixtures B and C, as shown in Fig. 5.

Besides binary Mixture A, we also studied the alignment characteristics of Compound (II). Similar results as those shown in Figs. 1-3 are observed.
In the LCD TVs employing PVA and MVA modes, the PI layers are needed but not rubbed. We also performed the alignment experiments of mixture A and Compound (II) using the same PI cells but without rubbing. The results were similar to those of buffed VA cells. Thus, our dopant-induced LC alignment improvement applies equally well to the rubbed and nonrubbed VA cells. We also applied our alignment method to the lateral difluoro-biphenyls. Similar improvements were obtained. Mixtures containing difluorobiphenyls and -terphenyls had $\Delta n \sim 0.2$ and excellent photo and thermal stabilities. ${ }^{19}$ These mixtures will be useful for high-contrast LCD TVs.

The detailed mechanisms responsible for the observed poor VA of difluoro-terphenyls in the buffed PI cells is not yet completely understood, but is speculated due to the molecular interactions between the difluoro group and the phenyl ring of the PI layers. The difluoro group has a strong negative charge and could be repelled from the PI so that the first monolayer tilts at a large angle. Therefore, the pretilt angle of the VA cell is large, resulting in light leakage through the crossed polarizers and smeared threshold behavior. The positive and nonpolar dopants dilute the negative charges, and result in an excellent alignment.

In conclusion, we have developed a simple method for aligning lateral difluoro-biphenyls and -terphenyls. By doping $\sim 10 \%$ of a positive or nonpolar material to the host negative LC mixtures, the buffed PI cells exhibit an excellent VA which is stable throughout its nematic range. In addition, the FOM of the doped mixtures is increased. The tradeoff is the slightly increased threshold voltage. On the other hand, negative $\Delta \varepsilon$ materials can also be used for the same purpose but at a higher concentration. The advantage of using a negative $\Delta \varepsilon$ dopant is to preserve the $|\Delta \varepsilon|$ of the host mixture. Useful application of this simple alignment method to LCD TVs is foreseeable.

${ }^{1}$ M. F. Schiekel and K. Fahrenschon, Appl. Phys. Lett. 19, 391 (1971). ${ }^{2}$ F. J. Kahn, Appl. Phys. Lett. 22, 386 (1973).

${ }^{3}$ A. Takeda, S. Kataoka, T. Sasaki, H. Chida, H. Tsuda, K. Ohmuro, T. Sasabayashi, Y. Koike, and K. Okamoto, SID Int. Symp. Digest Tech. Papers 29, 1077 (1998).

${ }^{4}$ Y. Ishii, S. Mizushima, and M. Hijikigawa, SID Int. Symp. Digest Tech. Papers 32, 1090 (2001).

${ }^{5}$ S. Kim, SID Int. Symp. Digest Tech. Papers 35, 760 (2004).

${ }^{6} \mathrm{~S}$. T. Wu and D. K. Yang, Reflective Liquid Crystal Displays (Wiley, New York, 2001).

${ }^{7}$ V. Reiffenrath, J. Krause, H. J. Plach, and G. Weber, Liq. Cryst. 5, 159 (1989).

${ }^{8}$ Y. Goto, T. Ogawa, S. Sawada, and S. Sugimori, Mol. Cryst. Liq. Cryst. 209, 1 (1991).

${ }^{9}$ G. W. Gray, M. Hird, and K. J. Toyne, Mol. Cryst. Liq. Cryst. 204, 43 (1991).

${ }^{10}$ D. Pauluth and K. Tarumi, J. Mater. Chem. 14, 1219 (2004).

${ }^{11}$ J. Cognard, Mol. Cryst. Liq. Cryst. Suppl. Ser. 1, 1 (1982).

${ }^{12}$ J. L. Janning, Appl. Phys. Lett. 21, 173 (1972).

${ }^{13}$ C. H. Wen, S. Gauza, J. Li, H. Wang, and S. T. Wu, Liq. Cryst. 32, 643 (2005).

${ }^{14}$ A. M. Lackner, J. D. Margerum, L. J. Miller, and W. H. Smith, Proc. SID 31, 321 (1990).

${ }^{15}$ W. H. Smith, H. L. Garvin, K. Robinson, and L. J. Miller, U.S. Patent No. $5,620,755$ (1997).

${ }^{16}$ M. Lu, K. H. Yang, T. Nakasogi, and S. J. Chey, SID Int. Symp. Digest Tech. Papers 31, 446 (2000).

${ }^{17}$ S. T. Wu, U. Efron, and L. D. Hess, Appl. Opt. 23, 3911 (1984).

${ }^{18}$ S. T. Wu, A. M. Lackner, and U. Efron, Appl. Opt. 26, 3441 (1987).

${ }^{19}$ S. Gauza, C. H. Wen, B. Tan, and S. T. Wu, Jpn. J. Appl. Phys., Part 1 43, 7176 (2004). 